\title{
Protective Effects of Ulinastatin Combined with Dexme- detomidine on Patients Undergoing One Lung Ventilation
}

\begin{abstract}
ZAIJING FAN, JIALE TIAN¹, YI CHEN² AND HUIJUN JIANG*
Department of Laboratory Medicine, Huzhou Central Hospital, Affiliated Central Hospital of Huzhou Normal University, Huzhou 313003, ${ }^{1}$ Department of Laboratory Medicine, Shanghai Tongji Hospital, Shanghai 200065, ${ }^{2}$ Department of Respiration, Huzhou Central Hospital, Affiliated Central Hospital of Huzhou Normal University, Huzhou 313003, P.R. China
\end{abstract}

Fan et al.: Combined Effects of Ulinastatin and Dexmedetomidine on OLV Patients

\begin{abstract}
To evaluate the protective effects of ulinastatin combined with dexmedetomidine on patients undergoing one lung ventilation. A total of 100 patients who underwent thoracoscopic partial lobectomy in our hospital were selected and randomly divided into control group (Group N), ulinastatin pretreatment group (Group U), dexmedetomidine continuous pumping group (Group D) and combined treatment group (Group U+D) $(n=25)$. Their intraoperative data and objective indices reflecting the lung function at $\mathrm{T} 1$, $\mathrm{T} 2$ and $\mathrm{T} 3$ were compared. The expression levels of inflammatory factors were compared at T0, T1, T2, T3 and T4. Lung dynamic compliance and oxygenation index rose first and then declined, while respiratory index declined first and then rose in all patients from $\mathrm{T} 1$ to $\mathrm{T} 3$. At T3, lung dynamic compliance and oxygenation index were significantly higher, while respiratory index was significantly lower in Group $U+D$ than those in the other groups $(\mathbf{p}<\mathbf{0 . 0 5})$. lung dynamic compliance, oxygenation index and respiratory index had significant differences in Group $U$ and D compared with those in Group N, but they had no significant differences between Group $U$ and $D(p>0.05)$. The levels of inflammatory factors rose first and then declined in all patients from T0 to T4. At T3 and T4, the levels of pro-inflammatory factors were significantly lower, while the level of anti-inflammatory factor was significantly higher in Group $U+D$ than those in the other groups $(\mathbf{p}<\mathbf{0 . 0 5})$. The levels of inflammatory factors had significant differences in Group U and D compared with those in Group $\mathbf{N}(\mathbf{p}<0.05)$, but they had no statistically significant differences between Group $U$ and $D(p>0.05)$. Group $U+D$ had a significantly lower incidence rate of postoperative pulmonary complications and a significantly shorter length of stay after surgery than those of Group U, D and $N$ $(p<0.05)$. Dexmedetomidine and ulinastatin can alleviate the damage caused by one lung ventilation to the lung to a certain extent and the combination of them has the optimum effect.
\end{abstract}

Key words: Ulinastatin, dexmedetomidine, one lung ventilation, lung protection

One lung ventilation (OLV) is an airway management method, in which special bronchial catheters are used to separate the ventilation paths of the two lungs during surgery and only the lung on the unaffected side is ventilated to make the lung on the affected side collapse. OLV keeps the affected lung completely closed, which not only expands the surgical field and operable space for surgeons, but also prevents the lung on the nonsurgical side from being infected by pulmonary secretions and blood on the surgical side. Therefore, OLV has been widely used in cardiac and thoracic surgeries. Despite many advantages and widespread application of OLV, its damage to the lungs is inevitable.

${ }^{*}$ Address for correspondence

E-mail: jhj19930410@yeah.net
During OLV, continuous mechanical ventilation and high tidal volume and respiratory rate are needed for the lung on the non-surgical side to realize the same effect as two-lung ventilation. Under such a breathing mode, the lung compliance will be altered, which can easily cause hypoxemia. If the tidal volume is too low, the oxygen supply cannot be guaranteed, easily leading to postoperative atelectasis ${ }^{[1]}$. Under such a nonphysiological state, the repeated collapse and reexpansion of the lung on the surgical side are likely to induce ischemia-reperfusion injury and stress response, so that the inflammatory factors are produced and released to further worsen the lung injury ${ }^{[2,3]}$. Currently, the lungs can be protected by anesthetic drugs to 
alleviate the damage of OLV to the lungs ${ }^{[4]}$. Therefore, the protection against OLV-related lung injury is a challenge encountered by anesthetists. Recently, ulinastatin (UTI) has been applied to prevent inflammatory responses in the perioperative period and avoid tissue and organ damage, obtaining obvious effects $^{[5]}$. Continuous infusion of dexmedetomidine (Dex) during surgery till the end can improve the lung function during anesthesia ${ }^{[6]}$. In this study, therefore, UTI and Dex were combined as drug intervention for patients undergoing OLV before anesthesia induction. The changes in the objective indexes reflecting lung function and the levels of inflammatory factors at different time points were compared, and the incidence rate of postoperative pulmonary complications (PPCs) and postoperative length of stay were recorded, aiming to assess the protective effects of the combination of the two drugs on lung function. A total of 100 patients who underwent thoracoscopic lobectomy in our hospital from January 2019 to January 2020 were selected and divided into control group (Group N), UTI pretreatment group (Group U), Dex continuous pumping group (Group D), and combined treatment group (Group $\mathrm{U}+\mathrm{D})(\mathrm{n}=25)$ using a random number table (Table 1$)$. Inclusion criteria: Patients with ASA grade I or II; those undergoing OLV during surgery within $2 \mathrm{~h}$ and anesthesia induction without repeated tracheal intubation; those without dysfunction of other organs, or underlying diseases such as diabetes and hypertension. Exclusion criteria: Patients with lung infection within 2 weeks before surgery; those who took drugs impairing the lung function for a long time; those with severe asthma. After entering the operating room, all patients were monitored for the heart rate, electrocardiogram and blood pressure. Midazolam injection (i.v., $0.05 \mathrm{mg} /$ $\mathrm{kg}$ ), fentanyl citrate injection $(3 \mu \mathrm{g} / \mathrm{kg})$, etomidate injection $(0.15 \mathrm{mg} / \mathrm{kg})$ and rocuronium bromide injection $(0.6 \mathrm{mg} / \mathrm{kg})$ were used for anesthesia induction. OLV was performed as follows. After the patient lost consciousness, the muscle relaxed and the blood pressure and heart rate became stable, a cannula was inserted trans-orally and a bronchial occluder (inner diameter: \#8) was placed. The correct position of occluder was confirmed by a fiberoptic bronchoscope, direct observation and auscultation, and then an anesthesia machine (Drager Fabius Tiro, Germany) was connected. The patients in each group received volumecontrolled mechanical ventilation and the respiratory parameters were adjusted according to the patient's weight: tidal volume (VT): $8 \mathrm{~mL} / \mathrm{kg}$, inspiratoryexpiratory ratio (I:E): $1: 2$, respiratory rate (RR): 12 breaths/min, fraction of inhaled oxygen (FiO2): 100\%, and oxygen flow rate: $2 \mathrm{~L} / \mathrm{min}$. By using a thoracoscope, the artificial pneumothorax was established by the surgeon, then OLV was started on the non-surgical side and the related respiratory parameters to the volume control of anesthesia machine were adjusted: VT: $6 \mathrm{~m} /$ $\mathrm{kg}, \mathrm{RR}: 14$ breaths/min and the remaining parameters were the same as above. Besides, sevoflurane was continuously inhaled to maintain anesthesia, its endexpiratory concentration was regulated around 1 Minimum alveolar concentration (MAC) and the cerebral state index of patients was kept at 40-60. After surgery, a chest drainage pipe was placed, and then two-lung ventilation was restored. Before anesthesia induction, $100 \mathrm{~mL}$ of $\mathrm{NaCl}$ mixed solution containing UTI (200 $000 \mathrm{U}$ ) was intravenously infused for Group U. For Group D, $\mathrm{NaCl}$ mixed solution containing Dex injection $(2 \mu \mathrm{g} / \mathrm{mL})$ was intravenously pumped continuously at $1 \mu \mathrm{g} /(\mathrm{kg} \bullet \mathrm{h})$ and it was intravenously pumped at $0.5 \mu \mathrm{g} /(\mathrm{kg} \bullet \mathrm{h})$ after anesthesia induction till the end of surgery. For Group U+D, $50 \mathrm{~mL}$ of $\mathrm{NaCl}$ mixed solution containing UTI (200 $000 \mathrm{U})$ and $50 \mathrm{~mL}$ of $\mathrm{NaCl}$ mixed solution $(4 \mu \mathrm{g} / \mathrm{mL})$ containing Dex injection $(2 \mu \mathrm{g} / \mathrm{mL})$ were added through the venous access for Group D. For Group N, $100 \mathrm{~mL}$ of $0.9 \%$ $\mathrm{NaCl}$ injection was intravenously infused. Five monitoring time points were set: before drug administration (T0), immediately after OLV (T1), 30 min after OLV (T2), completion of surgery (T3) and 12 $\mathrm{h}$ after surgery (T4). The lung dynamic compliance (Cdyn), oxygenation index (OI) and respiratory index (RI) were determined at T1, T2 and T3. The levels of inflammatory factors [interleukin-2 (IL-2), IL-6, IL-10 and tumor necrosis factor- $\alpha(\mathrm{TNF}-\alpha)$ ] were detected by enzyme-linked immunosorbent assay (ELISA) at T0, $\mathrm{T} 1, \mathrm{~T} 2, \mathrm{~T} 3$ and T4. In addition, the intraoperative data were recorded, including OLV time, anesthesia time, surgical time, blood transfusion volume, infusion volume and urine volume. The patients were followed up for $72 \mathrm{~h}$ after surgery, and the incidence of PPCs and postoperative length of stay were recorded. All data were statistically analyzed by statistical package for the social sciences (SPSS) 20.0 software. The quantitative data were expressed as $\overline{\mathrm{x}} \pm \mathrm{s}$ and subjected to one-way analysis of variance or $t$ test. The qualitative data were represented as rate and subjected to the Fisher's exact test or Chi-square test. $\mathrm{p}<0.05$ was considered statistically significant. There were no statistically significant differences in the intraoperative data including OLV time, anesthesia time, surgical time, blood transfusion volume, transfusion volume and 
urine output among the 4 groups $(\mathrm{p}>0.05)$ (Table 2). Cdyn and OI rose first and then declined, while RI declined first and then rose in all patients from $\mathrm{T} 1$ to $\mathrm{T} 3$. At T2, Cdyn and OI of Group U+D were significantly higher than those of Group N, and RI was significantly lower $(\mathrm{p}<0.05)$. At T3, Cdyn and OI were significantly higher, while RI was significantly lower in Group U+D than those in the other groups $(\mathrm{p}<0.05)$. Cdyn, OI and RI had significant differences in Group $U$ and $D$ compared with those in Group N, but they had no significant differences between Group U and D ( $p>0.05)$ (Table 3). The levels of inflammatory factors rose first and then declined in all patients from T0 to T4. At T2, the levels of pro-inflammatory factors (IL-2, IL-6, TNF- $\alpha$ ) in Group U+D were significantly lower than those of Group $\mathrm{N}$ and the level of anti-inflammatory factor (IL-10) was significantly higher $(\mathrm{p}<0.05)$. At T3 and $\mathrm{T} 4$, the levels of pro-inflammatory factors (IL-2, IL-6 and TNF- $\alpha$ ) were significantly lower, while the level of anti-inflammatory factor (IL-10) was significantly higher in Group $\mathrm{U}+\mathrm{D}$ than those in the other groups $(\mathrm{p}<0.05)$. The levels of inflammatory factors had significant differences in Group $\mathrm{U}$ and $\mathrm{D}$ compared with those in Group N $(\mathrm{p}<0.05)$, but they had no significant differences between Group $U$ and D $(\mathrm{p}>0.05)$ (Table 4). Group U+D had a significantly lower incidence rate of PPCs and a significantly shorter length of stay after surgery than Group U, D and N $(p<0.05)$. The incidence of PPCs and postoperative hospital stay in Group U and D were significantly lower than those of Group N ( $<<0.05)$, but Group U and D had similar results $(\mathrm{p}>0.05)$ (Table 5).

At present, the mechanism of lung injury during OLV remains controversial. According to the latest research, the inflammatory response plays an important role in the pathogenesis of lung injury. Therefore, the protective effect of Dex combined with UTI on the lungs of patients undergoing OLV was investigated through the objective indexes reflecting the lung function (Cdyn, $\mathrm{OI}$ and RI), the levels of plasma inflammatory factors (IL2, IL-6, IL-10 and TNF- $\alpha$ ) during inflammation, the incidence rate of PPCs and postoperative length of stay. Dex, a new type of highly-selective $\alpha-2$ adrenergic receptor agonist, is considered as a safe and feasible adjuvant drug for clinical anesthesia. Dex can downregulate the expression of pro-inflammatory factors and up-regulate the expression of anti-inflammatory factors, thereby exerting a protective effect on the lungs ${ }^{[7,8]}$. Chen et al. found that Dex alleviated the lung inflammation and improved the lung function through affecting the oxygenation state and Cdyn and inhibiting inflammatory factors ${ }^{[9]}$. UTI is a protease inhibitor extracted from human urine, which can exert an antiinflammatory effect via inhibiting the hydrolysis of various enzymes ${ }^{[10]}$. It has been confirmed that UTI can reduce cardiopulmonary bypass-associated lung injury ${ }^{[11]}$. However, some scholars still argued that UTI has limited anti-inflammatory and anti-oxidative stress effects, and proposed the combination of Dex and $\mathrm{UTI}^{[12]}$. Tao et al. also confirmed that the combination of UTI and Dex greatly reduced the stress response of patients undergoing OLV lobectomy, thereby protecting the lungs ${ }^{[13]}$. Cdyn is an index reflecting the elasticity of lung tissues and it can indicate the pathological changes in lung parenchyma more sensitively. A lower Cdyn means worse lung function $\left.{ }^{[14}\right]$. Both OI and RI are indexes reflecting the lung diffusion function, and they can assess the functional status of the lungs more accurately. The actual calculated value of RI is inversely proportional to the severity of lung injury ${ }^{[15]}$. OI is a diagnostic criterion for acute lung injury and acute lung injury can be diagnosed when the $\mathrm{OI}$ is 200$300^{[16]}$. In this study, Cdyn and OI significantly declined, while RI significantly rose in each group at T2 and T3 compared with those at $\mathrm{T} 1(\mathrm{p}<0.05)$, indicating that the lung function of patients declines and the lung diffusion function and tissue elasticity are affected. The results of comparison among groups revealed that Group U+D had significantly higher Cdyn and OI and significantly lower RI than Group N at T2. At T3, Cdyn, OI and RI in Group U+D also exhibited significant differences from those in Group $U$ and D $(p<0.05)$. Meanwhile, Cdyn, OI and RI in Group U and D had statistically significant differences compared with those in Group N, but they had no significant differences between Group $U$ and D $(p>0.05)$. It can be inferred that Dex or UTI intervention can alleviate the damage of OLV to the lungs and improve the lung function through affecting Cdyn, OI and RI, and the combination of the two drugs has the most significant effect before anesthesia induction. TNF- $\alpha$, IL-2 and IL- 6 are pro-inflammatory factors in the body and they are involved in the occurrence and development of lung inflammation ${ }^{[17,18]}$. Wang et al. observed and found in the rat model of lung ischemiaperfusion injury that IL-10, an anti-inflammatory factor, can reduce the pulmonary capillary permeability, thereby relieving lung injury ${ }^{[19,20]}$. Moreover, Zhao et al. found that the combined application of Dex and UTI can obviously enhance the lung function and reduce the levels of inflammatory factors ${ }^{[21]}$. The levels of inflammatory factors rose first and then declined in all patients from $\mathrm{T} 0$ to T4.At T2, the levels of pro- 
inflammatory factors (IL-2, IL-6, TNF- $\alpha$ ) in Group $\mathrm{U}+\mathrm{D}$ were significantly lower than those of Group $\mathrm{N}$ and the level of anti-inflammatory factor (IL-10) was significantly higher $(\mathrm{p}<0.05)$. At T3 and $\mathrm{T} 4$, the levels of pro-inflammatory factors were significantly lower, while the level of anti-inflammatory factor was significantly higher in Group $U+D$ than those in other groups ( $p<0.05)$. The levels of inflammatory factors had statistically significant differences in Group $U$ and D compared with those in Group N $(\mathrm{p}<0.05)$, but they had no statistically significant differences between Group $\mathrm{U}$ and D ( $>0.05)$. Group U+D had a significantly lower incidence rate of PPCs and a significantly shorter length of stay after surgery than Group U, D and N $(\mathrm{p}<0.05)$. Hence, OLV can affect the levels of anti-inflammatory and pro-inflammatory factors in vivo. Dex or UTI intervention before anesthesia induction has a certain inhibitory effect on inflammatory factors, avoiding the impact of the massive accumulation of inflammatory factors on the lung function. The combination of the two drugs has the optimal protective effect on the lungs. In conclusion, the combination of UTI and Dex exerts protective effects on the lungs of patients undergoing OLV, thus being worthy of promotion and application in clinical practice.

TABLE 1: BASELINE CLINICAL DATA

\begin{tabular}{|c|c|c|c|c|c|}
\hline Group & $U+D$ & $U$ & D & $\mathrm{N}$ & $P$ \\
\hline Age $(y)$ & $57.33 \pm 10.87$ & $56.42 \pm 10.25$ & $57.18 \pm 11.04$ & $56.13 \pm 11.12$ & 0.9748 \\
\hline $\begin{array}{l}\text { Gender (female/ } \\
\text { male) }\end{array}$ & $11 / 14$ & $9 / 16$ & $13 / 12$ & $12 / 13$ & 0.7022 \\
\hline BMI $\left(\mathrm{kg} / \mathrm{m}^{2}\right)$ & $21.54 \pm 2.13$ & $20.98 \pm 2.47$ & $21.38 \pm 2.34$ & $21.45 \pm 2.28$ & 0.0571 \\
\hline $\begin{array}{l}\text { Surgical site (left/ } \\
\text { right) }\end{array}$ & $10 / 15$ & $12 / 13$ & $11 / 14$ & $9 / 16$ & 0.8444 \\
\hline ASA grade (I/II) & $7 / 18$ & $9 / 16$ & $8 / 17$ & $9 / 16$ & 0.9194 \\
\hline
\end{tabular}

TABLE 2: INTRAOPERATIVE DATA

\begin{tabular}{|c|c|c|c|c|c|}
\hline Index & $U+D$ & $U$ & $\mathrm{D}$ & $\mathrm{N}$ & $P$ \\
\hline Surgical time (min) & $74.15 \pm 11.13$ & $78.15 \pm 10.97$ & $72.73 \pm 10.87$ & $73.76 \pm 10.39$ & 0.3130 \\
\hline $\begin{array}{l}\text { Anesthesia time } \\
(\mathrm{min})\end{array}$ & $87.76 \pm 10.17$ & $85.39 \pm 12.76$ & $88.13 \pm 11.74$ & $86.53 \pm 11.39$ & 0.7259 \\
\hline OLV time $(\min )$ & $52.47 \pm 8.97$ & $54.86 \pm 9.02$ & $53.42 \pm 9.18$ & $54.63 \pm 9.86$ & 0.7813 \\
\hline $\begin{array}{l}\text { Blood transfusion } \\
\text { volume }(\mathrm{ml})\end{array}$ & $87.15 \pm 11.19$ & $83.42 \pm 10.42$ & $84.17 \pm 10.94$ & $85.48 \pm 12.46$ & 0.6655 \\
\hline $\begin{array}{l}\text { Transfusion volume } \\
(\mathrm{ml})\end{array}$ & $594.57 \pm 45.63$ & $514.68 \pm 43.32$ & $525.29 \pm 40.17$ & $597.05 \pm 46.31$ & 0.4395 \\
\hline Urine output (ml) & $209.23 \pm 15.49$ & $215.46 \pm 14.12$ & $211.48 \pm 16.13$ & $207.84 \pm 13.67$ & 0.2960 \\
\hline
\end{tabular}

TABLE 3: OBJECTIVE LUNG FUNCTION INDICES

\begin{tabular}{lcccc}
\hline Index & Group & T1 & T2 & T3 \\
\hline \multirow{3}{*}{ Cdyn $(\mathrm{ml} / \mathrm{cmH} 20)$} & U+D & $34.25 \pm 4.53$ & $23.53 \pm 4.26^{(1)}$ & $30.87 \pm 3.97^{(1)(2)(3)}$ \\
& U & $33.86 \pm 4.37$ & $22.48 \pm 4.28$ & $28.87 \pm 4.21^{(1)}$ \\
& D & $34.18 \pm 4.16$ & $22.69 \pm 4.43$ & $27.63 \pm 4.08^{(1)}$ \\
& $\mathrm{N}$ & $34.02 \pm 3.94$ & $18.92 \pm 4.21$ & $24.39 \pm 3.81$ \\
OI $(\mathrm{mmHg})$ & $\mathrm{U}+\mathrm{D}$ & $378.58 \pm 21.93$ & $290.58 \pm 21.93^{(1)}$ & $348.58 \pm 21.93^{(1)(2)(3)}$ \\
& $\mathrm{U}$ & $371.81 \pm 22.42$ & $283.81 \pm 22.24$ & $335.81 \pm 21.42^{(1)}$ \\
& $\mathrm{D}$ & $373.76 \pm 22.33$ & $285.76 \pm 20.33$ & $334.76 \pm 20.33^{(1)}$ \\
& $\mathrm{N}$ & $375.56 \pm 21.59$ & $274.56 \pm 21.48$ & $327.56 \pm 20.59$ \\
$\mathrm{RI}$ & $\mathrm{U}+\mathrm{D}$ & $0.26 \pm 0.10$ & $0.96 \pm 0.33^{(1)}$ & $0.46 \pm 0.10^{(1)(2)(3)}$ \\
& $\mathrm{U}$ & $0.27 \pm 0.12$ & $1.18 \pm 0.34$ & $0.87 \pm 0.14^{(1)}$ \\
& $\mathrm{D}$ & $0.25 \pm 0.11$ & $1.05 \pm 0.30$ & $0.89 \pm 0.12^{(1)}$ \\
\hline
\end{tabular}

(1): Compared with Group N, p<0.05; (2): compared with Group U, p<0.05; (3): compared with Group D, $p<0.05$. 
TABLE 4: IL-2, IL-6, IL-10 AND TNF-A LEVELS AT DIFFERENT TIME POINTS

\begin{tabular}{|c|c|c|c|c|c|}
\hline Index & Group & T0 & $\mathrm{T} 2$ & T3 & T4 \\
\hline \multirow{4}{*}{$\mathrm{IL}-2(\mathrm{pg} / \mathrm{mL})$} & $U+D$ & $18.75 \pm 4.38$ & $23.47 \pm 4.61^{(1)}$ & $25.87 \pm 3.97^{(1)(2)(3)}$ & $20.47 \pm 4.23^{(1)(2)(3)}$ \\
\hline & $U$ & $18.69 \pm 3.72$ & $24.89 \pm 4.15$ & $28.87 \pm 4.21^{(1)}$ & $23.88 \pm 4.02^{(1)}$ \\
\hline & D & $18.43 \pm 3.91$ & $24.93 \pm 4.23$ & $27.63 \pm 4.08^{(1)}$ & $23.75 \pm 4.27^{(1)}$ \\
\hline & $\mathrm{N}$ & $19.07 \pm 4.25$ & $27.93 \pm 4.12$ & $34.87 \pm 3.92$ & $28.79 \pm 4.81$ \\
\hline \multirow{4}{*}{ IL-6 (pg/mL) } & $U+D$ & $21.98 \pm 4.93$ & $31.04 \pm 4.18^{(1)}$ & $37.03 \pm 3.65^{(1)}$ & $23.14 \pm 3.71^{(1)}$ \\
\hline & $U$ & $22.17 \pm 4.21$ & $33.57 \pm 4.53$ & $38.81 \pm 4.24^{(1)(2)(3)}$ & $27.39 \pm 3.85^{(1)(2)(3)}$ \\
\hline & $\mathrm{D}$ & $21.96 \pm 4.10$ & $32.76 \pm 4.16$ & $39.16 \pm 3.47^{(1)}$ & $27.68 \pm 4.03$ \\
\hline & $\mathrm{N}$ & $22.56 \pm 4.59$ & $38.11 \pm 4.32$ & $45.07 \pm 4.31$ & $29.17 \pm 3.76$ \\
\hline \multirow{4}{*}{$\mathrm{IL}-10(\mathrm{pg} / \mathrm{mL})$} & $U+D$ & $17.15 \pm 4.02$ & $21.06 \pm 4.27^{(1)}$ & $38.42 \pm 4.60^{(1)(2)(3)}$ & $28.24 \pm 4.53^{(1)(2)(3)}$ \\
\hline & U & $16.95 \pm 4.63$ & $19.83 \pm 4.77$ & $32.83 \pm 4.45^{(1)}$ & $24.36 \pm 4.08^{(1)}$ \\
\hline & $\mathrm{D}$ & $16.83 \pm 4.54$ & $19.59 \pm 4.30$ & $33.98 \pm 4.27^{(1)}$ & $23.97 \pm 4.19^{(1)}$ \\
\hline & $\mathrm{N}$ & $17.03 \pm 4.11$ & $16.72 \pm 4.85$ & $28.97 \pm 4.31$ & $21.83 \pm 4.17$ \\
\hline \multirow{4}{*}{ TNF-a (pg/mL) } & $\mathrm{U}+\mathrm{D}$ & $26.79 \pm 4.13$ & $30.86 \pm 4.31^{(1)}$ & $35.46 \pm 3.07^{(1)(2)(3)}$ & $28.87 \pm 3.76^{(1)(2)(3)}$ \\
\hline & U & $26.85 \pm 4.46$ & $32.73 \pm 3.25$ & $38.87 \pm 4.15^{(1)}$ & $33.78 \pm 3.52^{(1)}$ \\
\hline & $\mathrm{D}$ & $27.03 \pm 4.51$ & $32.52 \pm 3.03$ & $38.89 \pm 4.12^{(1)}$ & $32.89 \pm 4.83^{(1)}$ \\
\hline & $\mathrm{N}$ & $26.27 \pm 4.19$ & $35.49 \pm 4.26$ & $40.97 \pm 4.31$ & $35.71 \pm 4.31$ \\
\hline
\end{tabular}

(1): Compared with Group N, p<0.05; (2): compared with Group U, $p<0.05$; (3): compared with Group D, $p<0.05$.

TABLE 5: POSTOPERATIVE INDICES

\begin{tabular}{lcccc}
\hline Index & Group U+D & Group U & Group D & Group N \\
\hline $\begin{array}{l}\text { Incidence of PPCs (\%) } \\
\begin{array}{l}\text { Postoperative } \\
\text { hospitalization stay } \\
\text { (min) }\end{array}\end{array}$ & $6.32 \pm 0.58^{(1)(2)(3)}$ & $8.47 \pm 0.34^{(1)}$ & $9.07 \pm 0.42^{(1)}$ & $10.54 \pm 0.47$ \\
\hline
\end{tabular}

(1): Compared with Group N, p<0.05; (2): compared with Group U, $p<0.05$; (3): compared with Group D, $p<0.05$.

\section{Authors' contributions:}

Zaijing Fan and Jiale Tian are regarded as co-first authors.

\section{Conflicts of Interest:}

The authors declared no conflicts of interest.

\section{REFERENCES}

1. Luo Q. Effect of dexmedetomidine on lung compliance and oxygenation in elderly patients with one-lung ventilation anesthesia. Guangxi Med Univ 2019.

2. Ko YA, Yang MC, Huang HT, Hsu CM, Chen LW. NF-кB activation in myeloid cells mediates ventilator-induced lung injury. Respir Res 2013;14(1):1-3.

3. Kumar A, Pappu A, Sharma S, Sood J. Indigenous continuous positive airway pressure device for mitigation of hypoxemia during one lung ventilation. Anesth Analg 2016;123(6):1636.

4. Tao G, Chen Q, Feng D. Research progress in the protection of lung function in patients with one-lung ventilation. Mod $\mathrm{J}$ Integr Tradit Chin Western Med 2020;2:224-8.

5. Zhang W, Zhang J. Dexmedetomidine preconditioning protects against lung injury induced by ischemia-reperfusion through inhibition of autophagy. Exp Ther Med 2017;14(2):973-80.
6. Jiang YX, Shi-Yuan XU, Zhang XP. Protective effect of dexmedetomidine-ulinastatin combination on lipopolysaccharide-induced acute lung injury in rats. Chin J Pathophysiol 2014;30(1):96-101.

7. Zhang Q, Wu D, Yang Y, Liu T, Liu H. Dexmedetomidine alleviates hyperoxia-induced acute lung injury via inhibiting NLRP3 inflammasome activation. Cell Physiol Biochem 2017;42(5):1907-19.

8. Liuping WU, Cao S, Gao Y, Chen J. The protective effects of dexmedetomidine on lung injury during one lung ventilation in patients undergoing lobectomia pulmonalis. J Clin Anesth 2017;33(12):1171-3.

9. Chen W. Clinical significance of applying dexmedetomidine to patients with one-lung ventilation during thoracotomy. J Chin Foreign Med Res 2020;03:44-46.

10. Zhang L, Zhou XJ, Zhan LY, Wu XJ, Li WL, Zhao B, et al. Dexmedetomidine preconditioning protects against lipopolysaccharides-induced injury in the human alveolar epithelial cells. Rev Bras Anestesiol 2017;67(6):600-6.

11. Wang Y, Chen Y. Clinical analysis of the protective effect of ulinastatin on lung injury after cardiopulmonary bypass. Shaanxi Med J 2008;12:1620-2.

12. Kasper B, Salameh A, Krausch M, Kiefer P, Kostelka M, Mohr $\mathrm{FW}$, et al. Epigallocatechin gallate attenuates cardiopulmonary bypass-associated lung injury. J Surg Res 2016;201(2):313-25. 
13. Tao Y, Tao G, Wu W, Liu W, Li W, Zhang W, et al. The effect of dexmedetomidine combined with ulinastatin on stress response indexes of patients undergoing one-lung ventilation lobectomy. Chin Pharm 2017;28(35):4943-7.

14. Kong Y. Influence of pressure-regulated volume-controlled ventilation mode on the cardiopulmonary function of infants with complicated congenital heart disease after surgery. Chin Pract Med 2012;30:126-7.

15. Huang B. Clinical and experimental research on application of one-lung ventilation anesthesia in thoracic surgery. Guangxi Med Univ 2012.

16. Zhang ZQ, Chen RC, Yang QK, Li P, Wang CZ, Zhang ZH. A randomized controlled trial study of pulmonary rehabilitation with respiratory physiology as the guide on prognosis in patients with chronic obstructive pulmonary disease. Zhongguo Wei Zhong Bing Ji Jiu Yi Xue 2008;20(10):607-10.

17. Sun W. The effect of aminophylline on inflammatory indexes and lung function in children with cough variant asthma. Chin Mater Child Health Care 2013;24:3971-3.

18. Li D, Hu D, Li Y, Tai Y, Fang L, Yu Y, et al. Correlation between inflammatory cytokines and myocardial injury in early stages of sepsis. Anhui Med J 2014;12:1627-9.
19. Dai QC, Han Y, Miao XY. Effect of hydrogen sulfide on inflammatory cytokines in plasma and lung tissue of LPS induced acute lung injury rats. Chin J Gerontol 2014;34(7):1864-7.

20. Wang HL, Yi-Mou WU, Zheng JH. Study on the inflammatory injury of lung tissue and influence on inflammatory cytokines in mouse induced by recombinant protein CPAF from Chlamydophila pneumoniae. Chin J Immunol 2011;27(4):30811.

21. Zhao J, Che X. The protective effect of different doses of dexmedetomidine combined with ulinastatin on lung injury in patients undergoing unilateral lobectomy. Chin Pharm 2018;24:83-6.

This is an open access article distributed under the terms of the Creative Commons Attribution-NonCommercial-ShareAlike 3.0 License, which allows others to remix, tweak, and build upon the work non-commercially, as long as the author is credited and the new creations are licensed under the identical terms

This article was originally published in a special issue, "Clinical Research in Pharmaceutical and Biomedical Sciences" Indian J

Pharm Sci 2021:83(1)spl issue "219-224" 